\title{
ARTICLES
}

\section{Auxin transport is sufficient to generate a maximum and gradient guiding root growth}

\author{
Verônica A. Grieneisen ${ }^{1}$, Jian Xuㄹ ${ }^{2}$, Athanasius F. M. Marée ${ }^{1}$, Paulien Hogeweg ${ }^{1}$ \& Ben Scheres ${ }^{2}$
}

The plant growth regulator auxin controls cell identity, cell division and cell expansion. Auxin efflux facilitators (PINs) are associated with auxin maxima in distal regions of both shoots and roots. Here we model diffusion and PIN-facilitated auxin transport in and across cells within a structured root layout. In our model, the stable accumulation of auxin in a distal maximum emerges from the auxin flux pattern. We have experimentally tested model predictions of robustness and self-organization. Our model explains pattern formation and morphogenesis at timescales from seconds to weeks, and can be understood by conceptualizing the root as an 'auxin capacitor'. A robust auxin gradient associated with the maximum, in combination with separable roles of auxin in cell division and cell expansion, is able to explain the formation, maintenance and growth of sharply bounded meristematic and elongation zones. Directional permeability and diffusion can fully account for stable auxin maxima and gradients that can instruct morphogenesis.

The multitude of concentration-dependent effects of externally supplied auxins on plant development has long fuelled the idea that auxins form gradients in plants and generate developmental outputs $^{1-3}$. Auxin distributions at the cellular level can be inferred from the activity of auxin-responsive promoter elements. This led to the discovery that auxin-response maxima in roots, embryos and shoot-derived primordia require polar auxin transport, mediated by efflux-facilitating transmembrane proteins of the PIN family ${ }^{4-7}$. In shoots, auxin maxima predict sites of primordia outgrowth ${ }^{8,9}$ and leaf venation ${ }^{10}$; the role of auxin transport has been addressed in several modelling studies ${ }^{11-15}$. In the Arabidopsis root, an auxin response maximum is required for cell-type specification in its close vicinity, but also for cell division and cell expansion at a distance $e^{4,16}$. This stable auxin maximum has been assumed to necessarily involve, in addition to polar auxin transport, regulated auxin synthesis and/or breakdown, sources and/or sinks ${ }^{5}$, yet the essential mechanisms underlying its formation and maintenance remained unclear. Furthermore, auxin response maxima may not only reflect differential auxin concentrations but also local changes in auxin signal transduction components ${ }^{17,18}$. Here we provide a minimal mechanism for auxin-based pattern formation, given experimentally known PIN localizations. We develop and experimentally validate a mesoscopic model on the basis of the central assumption that PIN proteins have a critical role in polar auxin transport, which is supported by the dramatic defects of combinations of pin mutants ${ }^{7,16}$. The model reveals that the PIN-mediated flux pattern can explain where and why a root auxin maximum and associated auxin gradients arise as robust manifestations of the auxin transport system.

\section{A modelling framework for polar auxin transport}

Auxin diffuses freely within cells and the cell wall and permeates cell membranes ${ }^{19-21}$. Most previous simulation models at the tissue/organ scale have mathematically combined diffusion and permeability into one effective flux ${ }^{22}$. In contrast, by taking the spatial structure of cells into account ${ }^{23}$ in our model, diffusion and permeability are dealt with independently, using realistic parameter values (for parameter analysis see Supplementary Methods) rather than describing cells as units containing only one auxin concentration value with limited possible fluxes between units. At the next scale of organization, our model specifies individual cell sizes within a structured tissue layout that describes the entire root tip. It represents cells as extended regions on a lattice characterized by appropriately scaled sizes and shapes (Fig. 1), including surrounding cell walls. The interplay of phenomena that arise from the different scales (cell shape and neighbourhood within the tissue) is essential for understanding the emerging auxin distribution.

Auxin influx and efflux are treated differently according to the following biochemical considerations. Most extracellular auxin is protonated, and thus freely permeates into cells - a process which may additionally be facilitated by AUX/LAX family influx transporters ${ }^{24}$. In the cytoplasm, auxin is de-protonated such that free passage outwards is very slow; consequently, transport facilitators are necessary that locally permeate the membrane and facilitate auxin efflux. PIN proteins have cell-type-specific polar localizations in a manner that fully correlates with auxin transport routes as determined by auxin labelling, local auxin production and bioassays ${ }^{16,25,26}$, although other directional transporters may have additional roles ${ }^{27,28}$. Hence, in our model we specifically focus on the localization of the PINs (with permeability $P_{\mathrm{e}_{\text {pin }}}$ ). We also allow for significant influx $\left(P_{\mathrm{i}}\right)$ and little efflux $\left(P_{\mathrm{e}_{b g}}\right)$; the latter is mainly due to a background of ubiquitously expressed PINs in all cells.

Although the specific cell layout of the Arabidopsis thaliana root served as a guide for the model, we simplified this layout to capture the essential features of root architecture and PIN localization required for generating and maintaining an auxin maximum. Cells differ in size depending on their position in the meristematic (MZ) or elongation zone (EZ). Characteristic polar PIN localization is specified within four generalized tissue types: a central region represents 'vascular' tissue with basally located PINs; peripheral regions that express PIN predominantly at the apex correspond to the 'epidermal' tissues; between these tissues, a subclass of the vascular tissue-the single 'border' cell layer-has basal-lateral expression of PINs; and finally, the distal 'cap' region expresses PINs ubiquitously along the cell membrane (Fig. 1). In the epidermal cell layer, very low expression of lateral PINs is experimentally observed. Although lateral inward-localized PINs give no qualitative effect on the auxin distribution, they do generate quantitative effects (Supplementary Fig. 1). We thus introduce them when they become necessary for the dynamics of root 
growth. The results shown below are robust with respect to varying the number of cell files chosen for the layout (Supplementary Fig. 2). We first investigated critical features of the transport system in a static cellular system and subsequently analysed the full model, including growth, in which the formation of a meristematic and expansion zone arises as a self-organizing property given a pre-set response function for auxin-regulated cell division, growth and expansion.

\section{A polar-auxin-transport-dependent maximum}

Simulations with auxins provided from the uppermost vascular region reach a steady state with a strong concentration peak spanning several cells and with a maximum in cells immediately above the cap region (Fig. 2a, b), corresponding to the experimentally observed auxin response maximum in Arabidopsis quiescent centre cells. The model indicates that the appearance of the auxin maximum depends only on the general layout of PIN-mediated permeability, because specific cellular activities, such as localized decay of auxin levels or auxin influx regulation, are explicitly not included. Variation of permeability over a wide parameter range, which could reflect either different transport rates or different membrane densities of PINs, does not cause the maximum to disappear (Supplementary Fig. 3). The stable concentration peak is accompanied by large auxin fluxes through the tissues; like a reversed fountain, the central flow downwards connects to the upward flow in the epidermal tissue through a redistributing root cap (Fig. 2c). The auxin increase is due to reflux of the upward flow all along the meristem back into the central downward flow, which captures auxin within a flux-loop, causing an increase of the concentration maximum, until a steady state is reached (Fig. 2b). The overall PIN layout specifies the region of the maximum

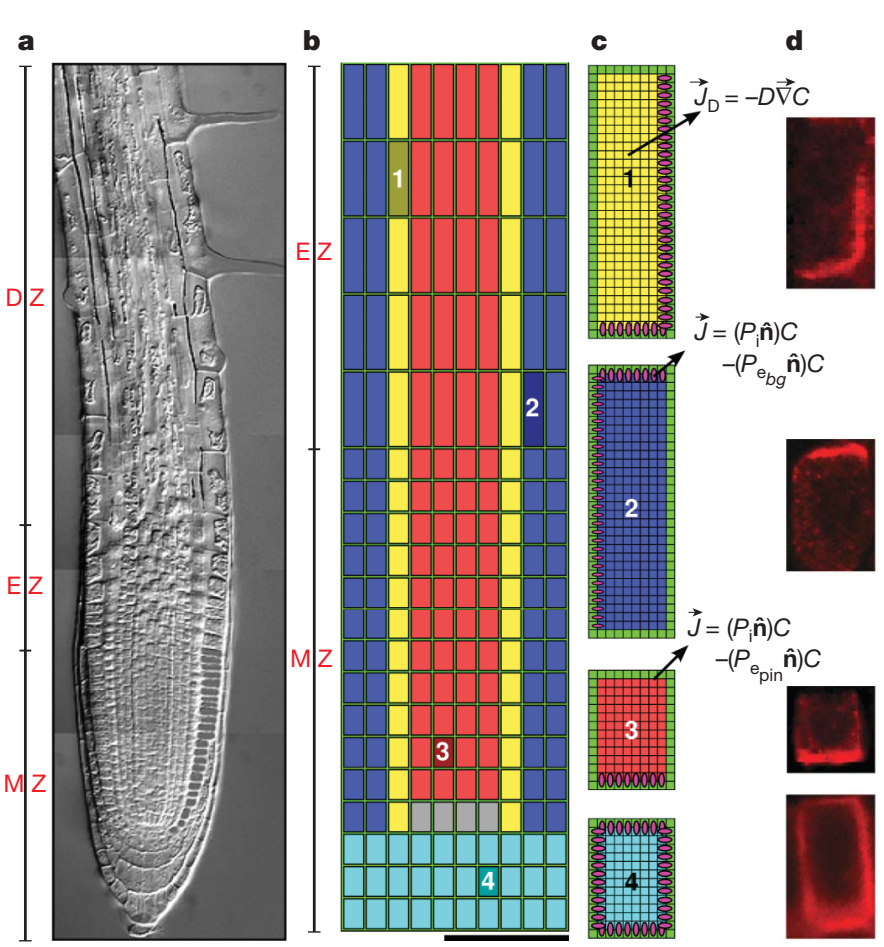

Figure 1 Mesoscopic model for polar auxin transport. a, The Arabidopsis root. DZ, differentiation zone; EZ, elongation zone; $\mathrm{MZ}$, meristematic zone. b, Generic root description adopted for the model. The four distinct regions treated in the simulations are indicated (vascular, red; epidermal layers, blue; border cells, yellow; and columella tiers, cyan). Quiescent centre (QC) cells (in grey) are equivalent to vascular cells. Scale bar, $100 \mu \mathrm{m}$. A cell wall/apoplast (green) surrounds all cells. Cells in the MZ and EZ have the same width,

$16 \mu \mathrm{m}$, but differ in length: $60 \mu \mathrm{m}$ (EZ) and $24 \mu \mathrm{m}$ (MZ). c, PIN localization within the cells that compose these tissues, based on experimentally observed PIN distributions (d). For simplicity, influx carriers AUX (considered ubiquitously expressed in every cell) are not shown. For an explanation of the equations used, see Methods Summary and Supplementary Methods. at the junction of the flows, which is positioned centrally just above the cap region. The density of lateral PINs, both those in the border and epidermal files (yellow and blue cells in Fig. 1), are crucial for the reflux and are the most sensitive determinants for the auxin-storage capacity of the root and the magnitude of the maximum (Supplementary Figs 1 and 3). The formation of the root's auxin maximum goes beyond the
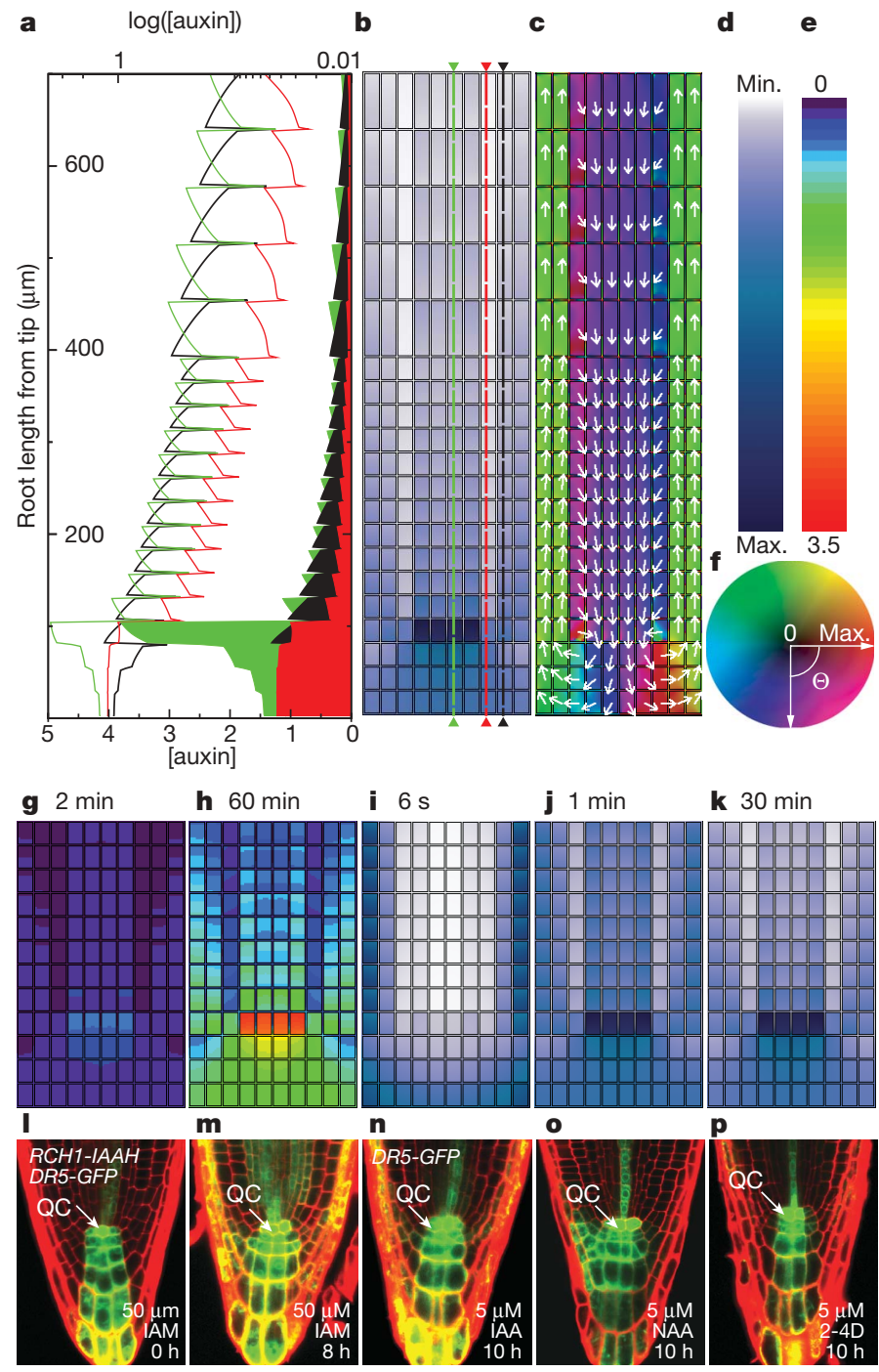

Figure 2 | Auxin maximum in simulations (a-k) and experiments (I-p).

a, The steady-state concentration profiles along longitudinal sections through the different tissues as indicated by coloured arrows in $\mathbf{b}$. Lines represent $\log ([$ auxin] $)$; filled profiles represent [auxin]; concentrations are in arbitrary units (A.U.), see Supplementary Methods 4.3. b, Steady-state concentrations within the root (Supplementary Movie 1), according to the colour bar (d) and scaled from the minimum (min.) to the maximum (max.) auxin concentration. c, Log of auxin fluxes measured in $\mu \mathrm{m}^{-1} \mathrm{~s}^{-1}$ (A.U.) forming the flux-reflux pattern. The directionality and magnitude of the fluxes are given by the colour (arc) and intensity (radius) as indicated by the colour circle (f), as well as by the arrows. Ubiquitous endogenous auxin production, simulated $(\mathbf{g}, \mathbf{h})$ or experimentally induced $(\mathbf{I}, \mathbf{m})$, is shown. The established pattern after $1 \mathrm{~min}(\mathrm{~g})$ and $60 \mathrm{~min}(\mathrm{~h})$ (Supplementary Movie 2) is shown. The absolute concentration values are shown according to the colour bar (e). DR5-GFP expression in roots of $\mathrm{RCH} 1-\mathrm{IAAH}$ lines that are ubiquitously biosynthesizing auxin at $0 \mathrm{~h}(\mathrm{I})$ and $8 \mathrm{~h}(\mathrm{~m})$ after induction by external addition of the precursor IAM $(50 \mu \mathrm{M})$. A normal auxin distribution is observed. Root immersion in auxin, simulated (i-k) and in experiments (n-p), is shown. Establishment of the pattern, according to the colour bar (d), $6 \mathrm{~s}(\mathbf{i}), 2 \mathrm{~min}(\mathbf{j})$ and $30 \mathrm{~min}(\mathbf{k})$ after immersion in auxin (Supplementary Movie 3, and Supplementary Methods for details). DR5-GFP expression in roots immersed in $5 \mu \mathrm{M} \mathrm{IAA} \mathrm{(n),} \mathrm{NAA}$ $(\mathbf{o})$, and $2-4 \mathrm{D}(\mathbf{p})$, all after $10 \mathrm{~h}$. All confocal images were taken using the same settings. In $\mathbf{g}-\mathbf{k}$ we show the most distal $314 \mu \mathrm{m}$ of the root. 
classical description of uni-directional transport causing accumulation $^{29}$, because it essentially depends on global features of PIN localization throughout the root that allow for reflux (Supplementary Fig. 4). High auxin throughput (up to $30 \%$ of the intracellular auxin per second) generates a stable but highly dynamic auxin equilibrium profile that requires neither production nor decay in the roots.

Auxin equilibrates into the given profile solely as a result of the high auxin flows and PIN localizations. When all cells ubiquitously produce auxin, the same pattern is generated (Fig. $2 \mathrm{~g}$, h). Even when production is localized outside the auxin-maximum region, the pattern does not change (Supplementary Fig. 5). To test experimentally the prediction that the auxin maximum is insensitive to a ubiquitous rise in auxin levels, we stimulated the production of the natural auxin indoleacetic acid (IAA) in the root meristem using the meristem-specific $\mathrm{RCH} 1$ promoter and the IAAH biosynthetic enzyme that can convert precursor IAM to IAA ${ }^{16,30}$. IAA levels were monitored with the semiquantitative and threshold dependent auxin response reporter DR5-GFP. As in the simulation, a persistent maximum is observed at the quiescent centre (Fig. 2l, m). We simulated an extreme case in which an auxin-free root lacking any influx from the shoot yet maintaining a normal efflux is immersed in an auxin solution. We find that auxin is polarly transported and rapidly redistributes in the immersed root tip, such that the auxin maximum is formed (Fig. 2i-k). Immersion of Arabidopsis roots in high concentrations of IAA indeed reveal the same persistent DR5GFP pattern (Fig. 2n), further supporting the notion that the auxin maximum in the root tip is insensitive to the position of the auxin source. To show that the auxin transport system is robust to permeability rates, as predicted by our simulations, immersion experiments were performed with the synthetic auxins, 2,4-dichlorophenoxyacetic acid (2,4-D) and 1-naphthylacetic acid (NAA). The resulting auxin analogue distributions indicate the preservation of the maximum (Fig. 2o, p). The auxin transport mechanism maintains the auxin maximum as long as the throughput exceeds the rate of uptake.

Our model predicts autonomy of the root system from the shoot; the auxin maximum remains stable even when the shoot, that is, the auxin supply, is removed. Simulating a sudden cessation of shootderived auxin into the root, we find that, although total levels of auxin may slowly drop, the pattern around the maximum remains highly conserved (Fig. 3a, b). To test this prediction, we dissected roots from shoots, and monitored auxin levels in the root tip by expression of the auxin sensors DR5-GFP and IAA2-GUS (Fig. 3c, d). In accordance with the modelling result, persistent expression of different reporters in the maximum is observed for more than 10-30 days after root cut, corroborating that the auxin transport system can maintain the maximum (Fig. 3e-j). Importantly, the amount of reflux in the root determines the timescales of auxin-maxima maintenance. Thus, the root is analogous to a capacitor: it efficiently stores auxin, and it will, in the absence of shoot auxin supply and the presence of leakage and decay, maintain the maximum while only slowly undergoing auxin loss (Supplementary Fig. 6). The capacitance depends on the generalized layout of PIN localization in the root tissue. Only certain alterations in PIN distribution, which change the generalized layout cause drastic modifications in the auxin distributions (Supplementary Fig. 4).

Realistic cell sizes and diffusion and transport rates in our model allow us to investigate the timescale involved with the creation and re-establishment of an auxin maximum. In the simulations, the establishment of a pattern occurs very fast, dictated by the high throughput. Simulations of a quiescent centre ablation experiment by elimination of the cells containing the auxin maximum reveals a new maximum within the time range of minutes, which from then on steadily increases (Fig. $4 \mathrm{a}-\mathrm{c}$, upper panels). Even in a simulated root cut experiment, a new maximum is established rapidly after quiescent centre ablation (Supplementary Fig. 7). To validate the fast timescales of auxin-maximum formation by the reflux loop, we laserablated the quiescent centre of a dissected root. A shift in auxin concentrations occurs within $3 \mathrm{~h}$ as measured by DR5-GFP, which includes the GFP maturation time. Thus, a new maximum rapidly arises from redistribution within the isolated root (Fig. $4 a-c$, lower panels). These experiments confirm that the timescale involved in establishing the typical auxin maximum is faster than the timescale of cell differentiation ${ }^{31}$, and they show that the auxin maximum can be re-patterned without shoot auxin input.

To explore the mechanisms by which auxin-maximum positioning is taking place, we analysed early modifications in the auxin distribution seen in the pin3;pin4;pin7 mutant by simulations and in the experimental root. In this triple mutant, we observe the auxin maximum shift towards the root cap together with a reduction in its intensity (Fig. 4e, lower panel). Generally, PIN activities in multiple pin mutant combinations are difficult to dissect owing to compensatory changes in gene expression. However, compensatory changes in PIN1 and PIN2 proteins in the pin3;pin4;pin7 mutant can be visualized and do not occur at early stages (Supplementary Fig. 8). We simulated the triple mutant by inferring from the membrane localization that the lack of PIN3, PIN4 and PIN7 results in an extreme reduction of efflux permeability in the columella tiers, as well as a reduction of both basalorientated permeabilities in the vascular region and lateral inwards permeabilities in the border cell file (see Fig. 4 legend). Our simulation results match with the experimentally observed basal dislocation of the auxin maximum and concentration drop (Fig. 4e, upper panel). We next questioned why these alterations take place. Experimentally, such an analysis is difficult because permeability manipulation of the separate regions is not trivial, owing to both distinct and overlapping expression domains of PINs 3, 4 and 7. However, in the model, we can explore independently the functional contribution of different regions. The new positioning of the maximum can be attributed to the significant reduction in efflux permeabilities within the columella cap, which now, instead of effectively redirecting the flow, accumulates auxin, dislocating the maximum distally (Fig. 4f). The decrease in magnitude of the dislocated maximum is not due to the reduction in vascular apical PIN expression, because the model shows that a
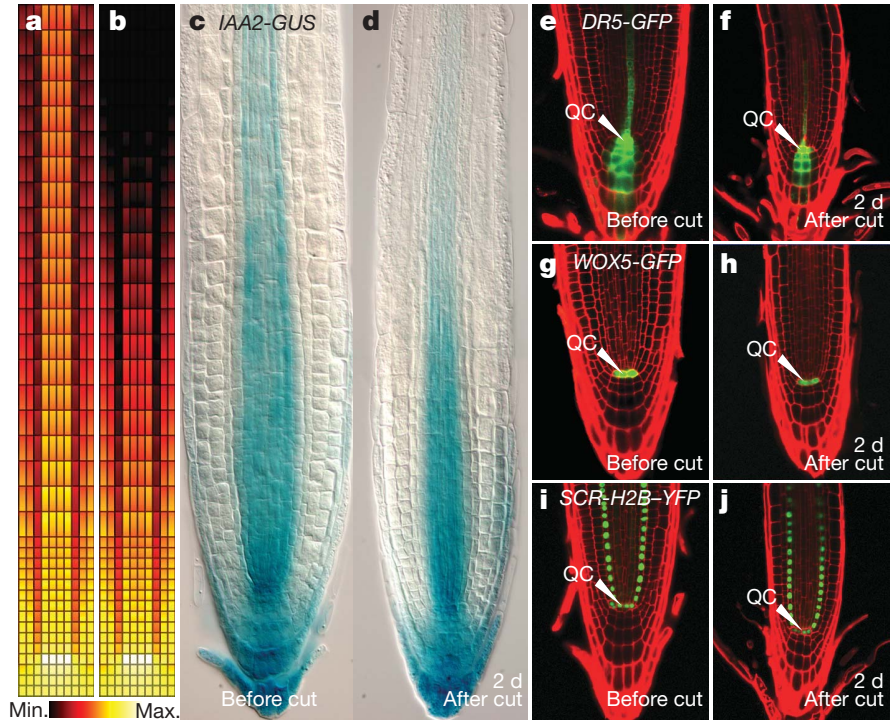

Figure 3 | An autonomous auxin capacitor in cut-root simulations (a, b) and experiments (c, d). a, Auxin distribution before cutting, that is, with auxin influx occurring at the uppermost vascular cells and efflux to the shoot occurring at the uppermost epidermal cells of a 1,700- $\mu \mathrm{m}$-long root. b, Auxin distribution after cutting, that is, after alteration of the top boundary conditions such that there is no influx of auxin (Supplementary Movie 4). Colours indicate the relative log of auxin concentrations, according to the colour bar below. c, d, IAA2-GUS expression in a four-day-old intact root (c), and $2 \mathrm{~d}$ after the root has been cut (d), revealing after the cut a persistent auxin maximum and gradient in its vicinity, and a decrease in auxin levels in the EZ. Expression patterns of DR5-GFP $(\mathbf{e}, \mathbf{f}), \operatorname{WOX5-GFP}(\mathbf{g}, \mathbf{h})$, and $S C R-$ $H 2 B-Y F P(\mathbf{i}, \mathbf{j})$, before $(\mathbf{e}, \mathbf{g}, \mathbf{i})$ and $2 \mathrm{~d}$ after $(\mathbf{f}, \mathbf{h}, \mathbf{j})$ root cut, revealing the correct maximum-associated gene-expression pattern. 
reduction of this transport alone should generally lead to a build up of auxin concentrations in the vascular region (Fig. 4g). Instead, the concentration reduction is in fact due to the decrease in capacitance caused by the reduced lateral PIN density (Fig. 4h). Combined, these alterations yield the mutant pattern.

Our simulations consistently reveal a characteristic exponential basipetal auxin gradient associated with the auxin maximum (Fig. 2a). Modelling higher lateral permeabilities of the border cells and/or epidermal cells reveals that this should lead to a steeper gradient as well as a higher maximum (Supplementary Figs 1-3). Interestingly, our model predicts that the stabilization of the maximum-associated gradient takes much longer than the formation of the maximum itself (Supplementary Fig. 9). This difference in timescale reflects that the steady state, in which the total auxin levels remain constant, is dictated by a large capacity to store auxin. While the root system receives auxin input, it rapidly passes through a transient phase in which the auxin maximum is formed. Once the maximum is established, the root enters a quasi-steady state (QSS) in which auxin levels increase but the auxin distribution profile maintains its overall shape, characterized by an exponential gradient culminating at the maximum, and a proximal flat concentration profile given by the shoot influx. The distal region of the gradient is, on a logarithmic plot, seen as a linear slope that connects to the flat concentration region. As the accumulation of auxin proceeds, the QSS evolves by the expansion of the exponential part of

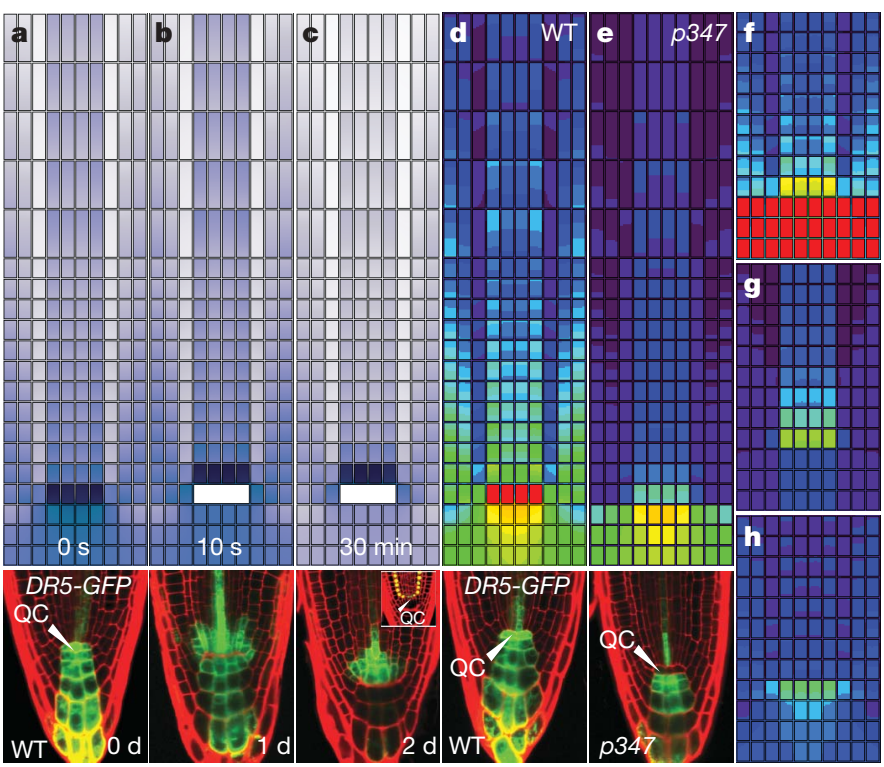

Figure 4 | Repositioning of the maximum owing to ablation and in the pin triple mutant, in simulations ( $a-e$, upper panels, and $f-h$ ) and experiments (a-e, lower panels). a-c, Ablation; dynamics of new maximum establishment after ablation of the QC in a cut root; upper panels, steady state immediately before cut and ablation (a, upper panel) and $10 \mathrm{~s}(\mathbf{b}$, upper panel) and $30 \mathrm{~min}$ (c, upper panel) after cut and ablation (Supplementary Movie 5). The simulated root is $1,700-\mu \mathrm{m}$ long, and here we show the most distal $700 \mu \mathrm{m}$. Colours are as shown in Fig. 1d. Lower panels, DR5-GFP expression before QC ablation (a, lower panel), 1 d after QC ablation ( $b$, lower panel), and $2 \mathrm{~d}$ after QC ablation (c, lower panel). QC ablations were performed $8 \mathrm{~h}$ after the root was cut. The inset in the lower panel of $\mathrm{c}$ shows $P S C R-H 2 B-Y F P$ expression $2 \mathrm{~d}$ after QC ablation, suggesting that QC re-specification has occurred. d-e, Triple mutant pin3;pin4;pin7 ( $p 347$ ) is simulated by reducing the permeability of the efflux values of vascular tissue and border cells to a quarter of their default values, and of columella tiers to a tenth of their default value. Default values and PIN orientations are as in Fig. 1 and Methods Summary. d, Wild-type auxin distribution. e, The resulting distribution in the triple mutant. Colours in upper panels of $\mathbf{d}$ and e give absolute concentration values according to the colour bar of Fig. 2e. d, Lower panel, DR5-GFP in WT. e, Lower panel, DR5-GFP;p347. f-h, Regional PIN contributions to the auxin distribution, with only root cap PIN reduction (f), only vascular PIN reduction $(\mathbf{g})$, or only lateral PIN reduction of border cells $(\mathbf{h})$. the profile into the proximal direction. In the absence of auxin decay, the system will practically always remain in the QSS, whereas with decay it remains in it for a period of days/weeks (dictated by the auxin half-life) before reaching equilibrium (Supplementary Fig. 9).

\section{Emergence and dynamics of zonation in growing roots}

We next considered the interplay between auxin dynamics and growth. We explore the pivotal issue of whether the auxin dynamics generated by our model produces developmentally instructive zonations (MZ, EZ) when simulating root growth. Auxin stimulates cell division and cell expansion at different concentrations in in vitro systems $^{32,33}$, and mutations in auxin response as well as in auxindependent patterning genes affect both cell division and cell expansion in roots ${ }^{34,35}$. Moreover, single or multiple pin mutants differentially affect cell division and cell expansion, further supporting spatially separable roles of auxin in the control of division and expansion zones ${ }^{16}$. As a simplifying approach we introduced a parsimonious description of the known auxin regulatory effects, that is, higher auxin levels promote cell division, whereas cell elongation is correlated with lower auxin levels. By implementing a graded response function that is identical for each cell and that dictates growth, division and rapid expansion (Fig. 5a, and Methods Summary for details), we explored whether growth feeds back on auxin distribution as well as if auxin concentrations alone could account for the formation of zonation. To capture possible slow developmental timescales, growth is simulated over an interval of 8 days.

At first observation, the growing root yields a similar auxin distribution to the static cell simulation, as long as the cellular growth rate does not yield a doubling time shorter than approximately 10-15 min, which is biologically unrealistic. The auxin maximum spans the same region during the growth process, maintaining itself in the correct position relative to the root tip (Fig. 5h, i). This robustness towards growth is due to the high auxin fluxes that accompany the maximum. Furthermore, our results reveal an emerging distal 'meristematic' region with small cells-and a proximal region where cell expansion prevails — caused by different levels of auxin within the self-generated auxin profile. Also in line with experimental data ${ }^{36}$, mitotic activity in vascular regions extends further up (Fig. 5e) than in peripheral regions. This difference is solely caused by auxin profile concentrations that are slightly higher within the vascular region.

Interestingly, the low density of lateral localized PINs in the epidermal cell files plays an unexpectedly important part in the robustness of the developmental response to the auxin gradient during growth. A more spread out and uneven auxin distribution occurs in the absence of lateral epidermal PINs (the lower capacitance case) than in their presence (Fig. 5b, d, respectively), bringing forth developmental changes. Visualization of mitotic density distributions, that is, the number of mitoses that occur relative to the distance from the quiescent centre, reveals that mitotic regions are more defined in roots with higher capacitance (Fig. 5e) than in the lower-capacitance case (Fig. 5c). The lateral epidermal PINs assure a sharper transition between the MZ and EZ. This creates a small distinct region close to the root tip in which cells are small (MZ), with a sigmoidal transition towards more elongated cells (EZ). The lower capacitance root, lacking the lateral epidermal PINs, under the same parameters, does not reveal a clear distinct zonation between small and large cells (Fig. 5f).

The size of the meristematic zone slowly increases over the simulated 8 days, as can be seen in the cell-size distributions (Fig. $5 \mathrm{~g}-\mathrm{i}$ ) and mitotic activities (Fig. 5j, k). This is due to the concentration profile being out of equilibrium (that is, in QSS), as can be inferred by the steadily increasing net auxin amount in the root system (inset of Fig. 51). Although the auxin slope is preserved, the transition between the zones becomes sharper, as can be seen by comparing the mitosis profiles at $12 \mathrm{~h}$ and 8 days (Fig. 5j, k). The increasing sharpness is due to feedback between the dynamics of cell growth and the gradient: while the number of meristematic cells rises, the rate of root growth increases, thereby speeding up the dislocation of the accompanying 
auxin gradient. Consequently, a cell at the transition region experiences a more dramatic decline in concentration, which, combined with the cells' graded auxin response, causes a sharpening of the transition. The slow timescale growth process observed in the

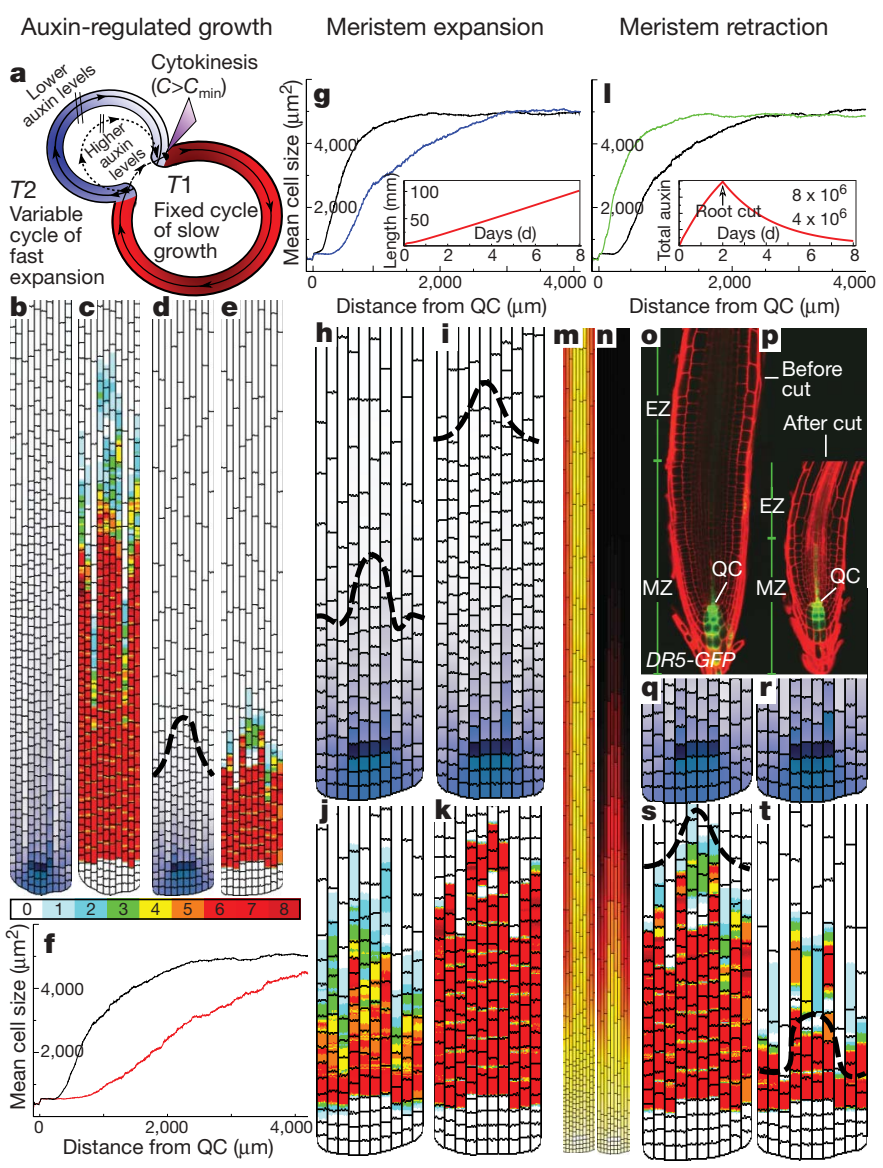

Figure 5 | Root growth and zonation in simulations (a-n, q-t) and experiments $(0, p)$. a, Model for auxin-regulated cell growth and expansion (see Methods Summary and Supplementary Methods). b-e, Selforganization of the MZ and EZ; auxin distribution and meristematic distribution of cell divisions after $1 \mathrm{~d}$ of growth without $(\mathbf{b}, \mathbf{c})$ and with (d, e) lateral efflux permeability at the epidermal cell layers, determining lower- and higher-capacitance roots, respectively. Colours in $\mathrm{c}$ and $\mathrm{e}$ indicate the number of mitotic events over a $12 \mathrm{~h}$ interval that occurred during the growth at that position relative to the distance from the QC, with colour coding indicated below. $f$, Average cell size as a function of the distance from the QC at $1 \mathrm{~d}$ of growth, of both a lower- (without lateral PINs in the epidermis; red line) and a higher- (with lateral PINs in the epidermis; black line) capacitance root. g, Expansion of the MZ; spatiotemporal averages of cell size, showing the expansion of the meristematic zone between $12 \mathrm{~h}$ (black line) and $8 \mathrm{~d}$ (blue line). Inset shows total length grown with time revealing an almost constant, slowly increasing growth rate over $8 \mathrm{~d}$. $\mathbf{h}, \mathbf{i}$, Auxin distribution and cell layouts at $12 \mathrm{~h}$ and $8 \mathrm{~d}$ of growth; $\mathbf{j}, \mathbf{k}$, the same MZ expansion seen through mitosis activity at the same time points (Supplementary Movie 6). I, Root cut and contraction of MZ;

spatiotemporal cell size distributions at the moment of root cut ( $2 \mathrm{~d}$; black line), and $6 \mathrm{~d}$ (green line) thereafter, showing meristem zone contraction after root cut. Inset reveals total auxin amount in a growing root receiving input from the shoot (first 2 days), and after root cut. $\mathbf{m}, \mathbf{n}$, Auxin distribution at the moment $(\mathbf{m})$ and $6 \mathrm{~d}$ after $(\mathbf{n})$ root cut, over the entire length of simulated root (Supplementary Movie 7). Colouring represents the $\log$ of auxin concentrations. o, p, DR5-GFP expression before (o) and $6 \mathrm{~d}$ after (p) root cut, showing conservation of the auxin maximum, and extension of the EZ into the MZ. $\mathbf{q}, \mathbf{r}$, Detailed images revealing auxinmaximum conservation: auxin distribution at the root tip at the moment of root cut $(\mathbf{q})$ and $6 \mathrm{~d}$ thereafter $(\mathbf{r}) . \mathbf{s}, \mathbf{t}$, Mitosis activity at the moment of root cut (s), and $6 \mathrm{~d}$ thereafter $(\mathbf{t})$, showing that meristematic activity becomes restricted nearer to the QC area after root cut. Dashed guiding lines in $\mathbf{d}, \mathbf{h}, \mathbf{i}$ and $\mathbf{s}$ help to visualize zonation that is due to cell size. simulations is in accordance with the increase of $\mathrm{MZ}$ found in wild-type roots ${ }^{37}$.

Simulating the root cut in the full model, yields a concentration drop in the upper region of the gradient (Fig. $5 \mathrm{~m}, \mathrm{n}$ ), whereas typical distributions around the auxin maximum are not altered (Fig. 5q, r). After the root cut, the EZ/MZ boundary shifts (Fig. 51), owing to the decay-driven auxin gradient shift (seen by a drop in total auxin shown in the inset of Fig. 5l). We tested this prediction by monitoring cell elongation in dissected experimental roots, and, indeed, recorded slow EZ expansion at the expense of the MZ (Fig. 5o, p). This effect was independently obtained by inhibiting shoot-derived polar auxin transport in intact seedlings using agarose blocks containing the polar auxin transport inhibitor NPA, again suggesting that the expansion of the EZ is caused by a reduction of total auxin levels (Supplementary Fig. 10). Taken together, our results show that EZ/MZ zonation is dynamically changing both in intact and dissected roots, whereas the auxin distribution around the quiescent centre is highly stable. Thus, the experimentally observed PIN distribution is able to explain fast, robust auxin-maximum formation, as well as the slow changes in zonation. Such seemingly uncorrelated processes thus do not necessarily depend on additional regulatory mechanisms.

\section{Discussion}

Our model strongly supports the hypothesis that plant roots are able to generate, through a combination of their topological structure, internal PIN distribution and basic properties such as diffusion and permeability, an auxin maximum and a highly robust gradient with morphogenic properties. This auxin distribution is robust at several levels: it preserves its characteristics through a broad set of permeability values, it is stable towards auxin production and decay, it is robust against drastic alterations in influx and efflux (such as root cut and tissue ablation), and it is not destabilized by cell division and expansion. The auxin maximum is formed without local auxin production or decay and without regulated influx. Thus, quiescent centre biosynthesis or 'sink' tissues are not essential for auxin-maximum localization. Lateral permeability enables roots to store and efficiently redistribute auxin. This has implications for interpreting experiments that track biosynthesis, because most endogenous production of auxin rapidly accumulates by auxin transport into the maximum, which does not necessarily correspond to the region of (highest) production. Moreover, the generalized PIN distribution used here is able to explain the location of the quiescent centre, as well as a characteristic exponential gradient of the auxin distribution. In addition, our mesoscopic modelling method reveals that information about the local neighbourhood exists, not only as an absolute concentration per cell, but also as a concentration gradient within the cell (Fig. 2a). This feature might be linked to auxin-dependent polar attributes of cells, such as the outgrowth of root hairs at the basal end of epidermal cells ${ }^{38}$.

Our implementation of auxin-dependent cell division and cell expansion reveals that it is possible to establish functional zonations of plant organs using polar auxin transport only, as long as the root has a high-enough degree of 'capacitance', that is, sufficient reflux, to maintain a steep gradient of auxin. We consider it highly likely that auxin maxima in plants are associated with auxin gradients, even though technical limitations have hitherto prohibited direct visualization of such gradients. Indirect observations point to the existence of an auxin gradient. For example, the PLETHORA genes, which are expressed in conjunction with the root auxin maximum and require auxin responsive transcription factors for expression, influence distal cell fate, cell division and cell expansion ${ }^{35}$. Notably, PLT proteins form a functional concentration gradient that extends up to the elongation zone ${ }^{39}$, which makes them good candidates for representing a read-out of a root auxin gradient.

The basic principles that endow roots with the capacity to form a developmentally robust and information-rich auxin gradient contrast with classical notions on developmentally instructive gradients, 
which are generated by 'a source' at the location of the highest concentration with associated gradients attributed to diffusion and decay $^{29}$. In our model, production and decay are not critical for auxin distributions as long as they occur on a slower timescale than the rapid auxin throughput. Moreover, it also differs from the classical concept of a transport-driven gradient, in which the slope results from the 'heaping up' of a morphogen at a 'dead end' (that is, a low throughput sink). Instead, the maximum and gradient are a consequence of the global PIN topology within the root, which establishes a reflux pattern with a high throughput of auxin. We have also shown that, after the establishment of the gradient, auxin throughput remains high, even (or rather, especially) at the location of accumulation. As a consequence of the reflux, the system is able to maintain global information during dynamic processes such as growth. Moreover, in contrast to the classical image of a frozen morphogenic gradient, here we show that a quasi-steady-state can transfer enough information to maintain a stem-cell-associated maximum, meristem and elongation zone, whereas, on a very different timescale, this profile changes, causing long-term developmental alterations. This reveals the importance of envisioning morphogenic gradients as non-static, out-of-equilibrium systems. In conclusion, transportdependent dynamic auxin gradients constitute a powerful mechanism to generate developmental information.

\section{METHODS SUMMARY}

Static simulations of auxin transport (see Fig. 1). PINs and AUXs are located at the interfaces between cell and cell-wall sites, orientated opposite to one another ( $\hat{\mathbf{n}}$ the unit vector normal to the surface of the membrane, orientated inwards), with permeabilities $P_{\mathrm{e}_{\mathrm{pin}}}$ and $P_{\mathrm{i}}$ of $20 \mu \mathrm{m} \mathrm{s}^{-1}$. All cell membranes are given a small background efflux, $P_{\mathrm{e}_{b s}}$, to account for membrane spreading of efflux carriers, which is set to $1 \mu \mathrm{ms}^{-1}$. The low epidermal lateral permeability $P_{\mathrm{e}_{\mathrm{pin}}}$ in the epidermis (represented as 'smaller' PINs in Fig. 1c, 2) is, for simplicity, set to zero in all static simulations, but used in the growing root case, in which it has a lower value of $5 \mu \mathrm{m} \mathrm{s}^{-1}$. Auxin diffuses freely within cells and cell walls, with a diffusion coefficient of $D=600 \mu \mathrm{m}^{2} \mathrm{~s}^{-1}$.

Simulations of growing roots (see Fig. 5a). The growth phase is described by a constant doubling time, $T 1$; the expansion phase, with eightfold higher volume increases, is described by a variable duration, $T 2$, inversely related to auxin level, $C$, at the centre of mass of each cell. $T 2=\max \left(\alpha \ln \left(C^{*} / C\right), 0\right)$, where $C^{*}$ is the concentration above which cells only undergo the cycle $T 1$. After $T 1$ and $T 2$, cytokinesis occurs if $C>C_{\min } . \alpha$ is a sensitivity parameter. We include a cell-size check of minimal area $720 \mu \mathrm{m}^{2}$ for division, and a maximum cell area of $4,800 \mu \mathrm{m}^{2}$ above which cell expansion stops. Growth-related parameter values are derived from experimental data (Supplementary Material section 4.5) and set to: $T 1=2 \mathrm{~h}, C_{\min }=3, C^{*}=10, \alpha=6.2 \mathrm{~h}$. The auxin decay rate is $\delta_{\text {decay }}=5 \times 10^{-6} \mathrm{~s}^{-1}$. Cell dynamics (growth and division) have been simulated by a modified Cellular Potts Model (see Supplementary Methods) in which turgor pressures drive cell movement within files. Cells expand in a longitudinal direction only (thus we assume stiff vertical cell walls); no constraints on relative cell file movements are included. Divisions are always anticlinal. Columella and quiescent centre cells don't divide. All simulations were run over the interval of 8 days, corresponding to $6,912,000$ numerical time steps.

\section{Received 8 May; accepted 3 September 2007.}

1. Berleth, T., Scarpella, E. \& Prusinkiewicz, P. Towards the systems biology of auxintransport-mediated patterning. Trends Plant Sci. 12, 151-159 (2007).

2. Leyser, O. Auxin distribution and plant pattern formation: how many angels can dance on the point of PIN? Cell 121, 819-822 (2005).

3. Jones, A. M. Auxin transport: down and out and up again. Science 282, 2201-2203 (1998).

4. Sabatini, S. et al. An auxin-dependent distal organizer of pattern and polarity in the Arabidopsis root. Cell 99, 463-472 (1999).

5. Friml, J. et al. AtPIN4 mediates sink-driven auxin gradients and root patterning in Arabidopsis. Cell 108, 661-673 (2002).

6. Benková, E. et al. Local, efflux-dependent auxin gradients as a common module for plant organ formation. Cell 115, 591-602 (2003).

7. Friml, J. et al. Efflux-dependent auxin gradients establish the apical-basal axis of Arabidopsis. Nature 426, 147-153 (2003)

8. Reinhardt, D. et al. Regulation of phyllotaxis by polar auxin transport. Nature 426, 255-260 (2003)

9. Heisler, M. G. et al. Patterns of auxin transport and gene expression during primordium development revealed by live imaging of the Arabidopsis inflorescence meristem. Curr. Biol. 15, 1899-1911 (2005).
10. Scarpella, E., Marcos, D., Friml, J. \& Berleth, T. Control of leaf vascular patterning by polar auxin transport. Genes Dev. 20, 1015-1027 (2006).

11. Sachs, T. The control of the patterned differentiation of vascular tissues. Adv. Bot. Res. 9, 152-262 (1981)

12. Feugier, F. G., Mochizuki, A. \& Iwasa, Y. Self-organization of the vascular system in plant leaves: inter-dependent dynamics of auxin flux and carrier proteins. J. Theor. Biol. 236, 366-375 (2005).

13. De Reuille, P. B. et al. Computer simulations reveal properties of the cell-cell signaling network at the shoot apex in Arabidopsis. Proc. Natl Acad. Sci. USA 103, 1627-1632 (2006).

14. Jönsson, H., Heisler, M. G., Shapiro, B. E., Meyerowitz, E. M. \& Mjolsness, E. An auxin-driven polarized transport model for phyllotaxis. Proc. Natl Acad. Sci. USA 103, 1633-1638 (2006).

15. Smith, R. S. et al. A plausible model of phyllotaxis. Proc. Natl Acad. Sci. USA 103 , 1301-1306 (2006).

16. Blilou, I. et al. The PIN auxin efflux facilitator network controls growth and patterning in Arabidopsis roots. Nature 433, 39-44 (2005).

17. Kepinski, S. \& Leyser, O. The Arabidopsis F-box protein TIR1 is an auxin receptor. Nature 435, 446-451 (2005).

18. Dharmasiri, N., Dharmasiri, S. \& Estelle, M. The F-box protein TIR1 is an auxin receptor. Nature 435, 441-445 (2005).

19. Gutknecht, J. \& Walter, A. Transport of auxin (indoleacetic acid) through lipid bilayer membranes. J. Membr. Biol. 56, 65-72 (1980).

20. Goldsmith, M. H. M., Goldsmith, T. H. \& Martin, M. H. Mathematical analysis of the chemosmotic polar diffusion of auxin through plant tissues. Proc. Natl Acad. Sci. USA 78, 976-980 (1981)

21. Kramer, E. M. \& Bennett, M. J. Auxin transport: a field in flux. Trends Plant Sci. 11, 382-386 (2006).

22. Kramer, E. M. Computer models of auxin transport: a review and commentary (2007). J. Exp. Bot. advance online publication doi:10.1093/jxb/erm060 (12 April 2007).

23. Kramer, E. M. PIN and AUX/LAX proteins: their role in auxin accumulation. Trends Plant Sci. 9, 578-582 (2004).

24. Marchant, A. et al. AUX1 regulates root gravitropism in Arabidopsis by facilitating auxin uptake within root apical tissues. EMBO J. 18, 2066-2073 (1999).

25. Rashotte, A. M., Poupart, J., Waddell, C. S. \& Muday, G. K. Transport of the two natural auxins, indole-3-butyric acid and indole-3-acetic acid, in Arabidopsis. Plant Physiol. 133, 761-772 (2003)

26. Wiśniewska, J. et al. Polar PIN localization directs auxin flow in plants. Science 312, 883 (2006).

27. Geisler, M. et al. Cellular efflux of auxin catalyzed by the Arabidopsis MDR/PGP transporter AtPGP1. Plant J. 44, 179-194 (2005).

28. Terasaka, K. et al. PGP4, an ATP binding cassette P-glycoprotein, catalyzes auxin transport in Arabidopsis thaliana roots. Plant Cell 17, 2922-2939 (2005).

29. Wolpert, L. Positional information and the spatial pattern of cellular differentiation. J. Theor. Biol. 25, 1-47 (1969).

30. Casamitjana-Martínez, E. et al. Root-specific CLE19 overexpression and the sol1/2 suppressors implicate a CLV-like pathway in the control of Arabidopsis root meristem maintenance. Curr. Biol. 13, 1435-1441 (2003).

31. $\mathrm{Xu}$, J. et al. A molecular framework for plant regeneration. Science 311, 385-388 (2006).

32. Went, F. W. Growth, auxin, and tropisms in decapitated Avena coleoptiles. Plant Physiol. 17, 236-249 (1942).

33. Skoog, F. \& Miller, C. O. Chemical regulation of growth and organ formation in plant tissues cultured in vitro. Symp. Soc. Exp. Biol. 54, 118-130 (1957).

34. Lincoln, C., Britton, J. H. \& Estelle, M. Growth and development of the axr1 mutants of Arabidopsis. Plant Cell 2, 1071-1080 (1990).

35. Aida, M. et al. The PLETHORA genes mediate patterning of the Arabidopsis root stem cell niche. Cell 119, 109-120 (2004)

36. Beeckman, T., Burssens, S. \& Inzé, D. The peri-cell-cycle in Arabidopsis. J. Exp. Bot. 52, 403-411 (2001).

37. Beemster, G. T. S. \& Baskin, T. I. Analysis of cell division and elongation underlying the developmental acceleration of root growth in Arabidopsis thaliana. Plant Physiol. 116, 1515-1526 (1998).

38. Masucci, J. D. \& Schiefelbein, J. W. The rhd6 mutation of Arabidopsis thaliana alters root-hair initiation through an auxin- and ethylene-associated process. Plant Physiol. 106, 1335-1346 (1994).

39. Galinha, C. et al. PLETHORA proteins as dose-dependent master regulators of Arabidopsis root development. Nature doi:10.1038/nature06206 (this issue).

Supplementary Information is linked to the online version of the paper at www.nature.com/nature.

Acknowledgements A.F.M.M. and B.S. are supported by the Netherlands Organization for Scientific Research (NWO). We thank I. Blilou for experiments performed in the early phase of this study and discussions. We acknowledge

T. Berleth and K. Ljung for helpful comments.

Author Information Reprints and permissions information is available at www.nature.com/reprints. Correspondence and requests for materials should be addressed to B.S. (b.scheres@uu.nl). 